\title{
AVALIAÇÃO DO ACESSO À FISIOTERAPIA APÓS A ALTA HOSPITALAR EM INDIVÍDUOS COM ACIDENTE VASCULAR Cerebral
}

\section{ASSESSMENT OF ACCESS TO PHYSIOTHERAPY AFTER HOSPITAL DISCHARGE IN POST-STROKE PATIENTS}

\author{
Raquel Estauber de Miranda ${ }^{1}$, Débora Schmidt², Laíde Hanauer ${ }^{1}$, \\ Simone Rizzo Nique Peralles ${ }^{1}$, Vera Lúcia Widniczck Striebel ${ }^{1}$
}

\section{RESUMO}

Introdução: O Acidente Vascular Cerebral (AVC) é a principal causa de incapacidades neurológicas em adultos jovens e a reabilitação precoce é essencial para a recuperação desses pacientes. O objetivo deste estudo foi investigar o acesso aos serviços de fisioterapia após a alta hospitalar (AH) em indivíduos com AVC.

Métodos: Trata-se de um estudo de coorte incluindo indivíduos com AVC, que foram avaliados quanto à funcionalidade e incapacidade na $\mathrm{AH}$ e 60 dias após. Foi realizado contato telefônico após 30 e 60 dias da AH para verificar o seguimento da fisioterapia.

Resultados: Trinta e seis indivíduos com sequelas motoras foram avaliados na $\mathrm{AH}$ apenas $19 \%$ realizaram seguimento fisioterapêutico após 30 dias da $\mathrm{AH}$ e $39 \%$ após 60 dias. As principais barreiras encontradas, mesmo após 60 dias da $\mathrm{AH}$, foram as dificuldades burocrática de acesso aos serviços de saúde (55\%) e o tempo de espera $(41 \%)$ para iniciar o tratamento. A funcionalidade, avaliada pela Medida Internacional de Funcionalidade e pela escala Rankin, apresentou melhora significativa após 60 dias da alta, tanto nos indivíduos que realizaram, como naqueles que não realizaram acompanhamento fisioterapêutico $(p=0,001)$.

Conclusão: $\mathrm{O}$ acesso à fisioterapia após a alta hospitalar para os indivíduos com AVC foi deficiente na amostra estudada devido principalmente às barreiras burocráticas e longo tempo de espera.

Palavras-chave: Reabilitação; Acidente Vascular Cerebral; saúde pública

\section{ABSTRACT}

Introduction: Stroke is the leading cause of neurological damage in young adults, and early rehabilitation is essential for patient recovery. The objective of this study was to investigate the access to physiotherapy services after hospital discharge in post-stroke patients.

Methods: The present study is a cohort study including post-stroke patients who were evaluated regarding ability and disability at hospital discharge and 60 days later. Patients were contacted by phone to check physiotherapy engagement, after 30 and 60 days.

Results: A total of 36 individuals with mobility impairment were observed after hospital discharge, among which only $19 \%$ had started physiotherapeutic therapy after 30 days of discharge from the hospital, and $39 \%$ after 60 days. Major barriers included health care bureaucracy $(55 \%)$, and the wait to start treatment $(41 \%)$. Ability, measured by the Physical Abilities and Mobility Scale and the Rankin Scale, showed significant improvement 60 days after hospital discharge both in patients who underwent physical therapy and in those who did not $(p=0.001)$.

Clin Biomed Res. 2018:38(3):245-252

1 Curso de Fisioterapia, Centro Universitário Metodista (IPA). Porto Alegre, RS, Brasil.

2 Serviço de Fisioterapia, Hospital de Clínicas de Porto Alegre. Porto Alegre, RS, Brasil.

Autor correspondente: Débora Schmidt debora.schmidt87@gmail.com Hospital de Clínicas de Porto Alegre Rua Ramiro Barcelos, 2350. 90035-903, Porto Alegre, RS, Brasil. 
Conclusion: Access to physical therapy after hospital discharge for post-stroke patients was lacking in the sample studied, mainly due to bureaucratic barriers and long waiting time.

Keywords: Rehabilitation; stroke; public health

O Acidente Vascular Cerebral (AVC) é uma doença com importante impacto na saúde pública, sendo a principal causa de incapacidades neurológicas e de elevadas taxas de mortalidade no Brasil e no mundo' Nas últimas décadas o número de pessoas com o primeiro AVC aumentou em $68 \%$ assim como a taxa de sobrevivência para $84 \%^{2}$. Em 2030, estima-se que o mundo terá 70 milhões de sobreviventes ao AVC, dos quais $70 \%$ não retornarão ao trabalho e $30 \%$ necessitarão de auxílio para locomoção ${ }^{3}$.

A lesão cerebral decorrente do AVC pode resultar em deficiências motoras, distúrbios na fala e deglutição e até mesmo disfunções cognitivas, levando a dificuldades na execução das atividades de vida diária, bem como, restrições na participação social, perda de autonomia e não retorno às atividades laborais. Esses pacientes estão entre os maiores usuários de serviços de reabilitação, representando um grande impacto econômico para os serviços públicos de saúde ${ }^{4}$.

No Brasil, o modelo assistencial baseado na integralidade do cuidado prevê o atendimento dos pacientes com AVC nos serviços de atenção básica, serviços de urgência e emergência, unidades especializadas de assistência no âmbito hospitalar, unidades de reabilitação, cuidados ambulatoriais e reintegração social ${ }^{3}$.

A reabilitação deve iniciar o mais precocemente possível, ainda na fase hospitalar, sendo os primeiros três meses considerados primordiais na evolução dos pacientes ${ }^{5}$. Após a alta hospitalar $(\mathrm{AH})$, a continuidade da reabilitação é essencial para a recuperação funcional, o que pode ser prejudicado pela dificuldade de acesso dos usuários a serviços especializados ${ }^{6}$.

O presente estudo teve como objetivo investigar o acesso dos indivíduos com sequelas motoras decorrentes do AVC aos serviços de fisioterapia após a $\mathrm{AH}$ e identificar as principais barreiras e dificuldades encontradas neste acesso.

\section{MATERIAIS E MÉTODOS}

Trata-se de um estudo de coorte, prospectivo, realizado no Hospital Nossa Senhora da Conceição em Porto Alegre/RS, no período de fevereiro a julho de 2017. A pesquisa foi aprovada pelo Comitê de Ética e Pesquisa da instituição (número 1.289.490). Todos os participantes consentiram com o Termo de Consentimento Livre e Esclarecido (TCLE).
A amostra, obtida por conveniência, foi composta por indivíduos maiores de 18 anos com diagnóstico de AVC como motivo da internação. Foram excluídos, os que não apresentaram condições cognitivas (pontuação <20 no Mini Exame do Estado Mental Pontuação) ${ }^{7}$ ou de comunicação para consentir com - TCLE, aqueles sem sequelas motoras e os que não tinham a possibilidade de contato telefônico após a $\mathrm{AH}$.

Os pacientes foram avaliados na $\mathrm{AH}$ através da Escala de Medida de Independência Funcional (MIF) e da Escala de Rankin modificada ${ }^{8,9}$. Trinta dias após a $\mathrm{AH}$ foi realizado contato telefônico com os indivíduos ou seu representante legal para obter informações a respeito do encaminhamento para serviços de reabilitação, tempo transcorrido para o início da fisioterapia, frequência semanal da fisioterapia, local de realização dos atendimentos e meio de transporte utilizado.

Um novo contato telefônico foi realizado sessenta dias após a AH para verificar o seguimento da fisioterapia e neste momento também foram reaplicadas as escalas MIF e Rankin.

\section{Análise Estatística}

Variáveis contínuas foram descritas utilizando média \pm desvio padrão para aquelas com distribuição normal e mediana e intervalo interquartílico (percentis 25;75), para as com distribuição assimétrica e frequência absoluta e relativa para as variáveis categóricas. Para comparar os escores de MIF e Rankin, pré e pós, foi utilizado o Teste de Wilcoxon. Para comparar a variação (delta) de MIF entre pacientes que fizeram ou não fisioterapia, foi utilizado o Teste de Mann-Whitney. O programa utilizado foi o SPSS, versão 16.0. Foi considerado significante $p<0,05$.

\section{RESULTADOS}

Durante o período do estudo 117 indivíduos internaram com AVC isquêmico, destes, 39 que apresentavam déficit motor no momento da $\mathrm{AH}$ foram incluídos no estudo. A avaliação completa foi realizada em 36 indivíduos, conforme demonstrado no fluxograma da Figura 1. A média de idade da amostra estudada foi de 61 anos, a maioria eram homens caucasianos e viúvos. As características demográficas da amostra estão apresentadas na tabela 1. 


\section{7 indivíduos com AVC Isquêmico}

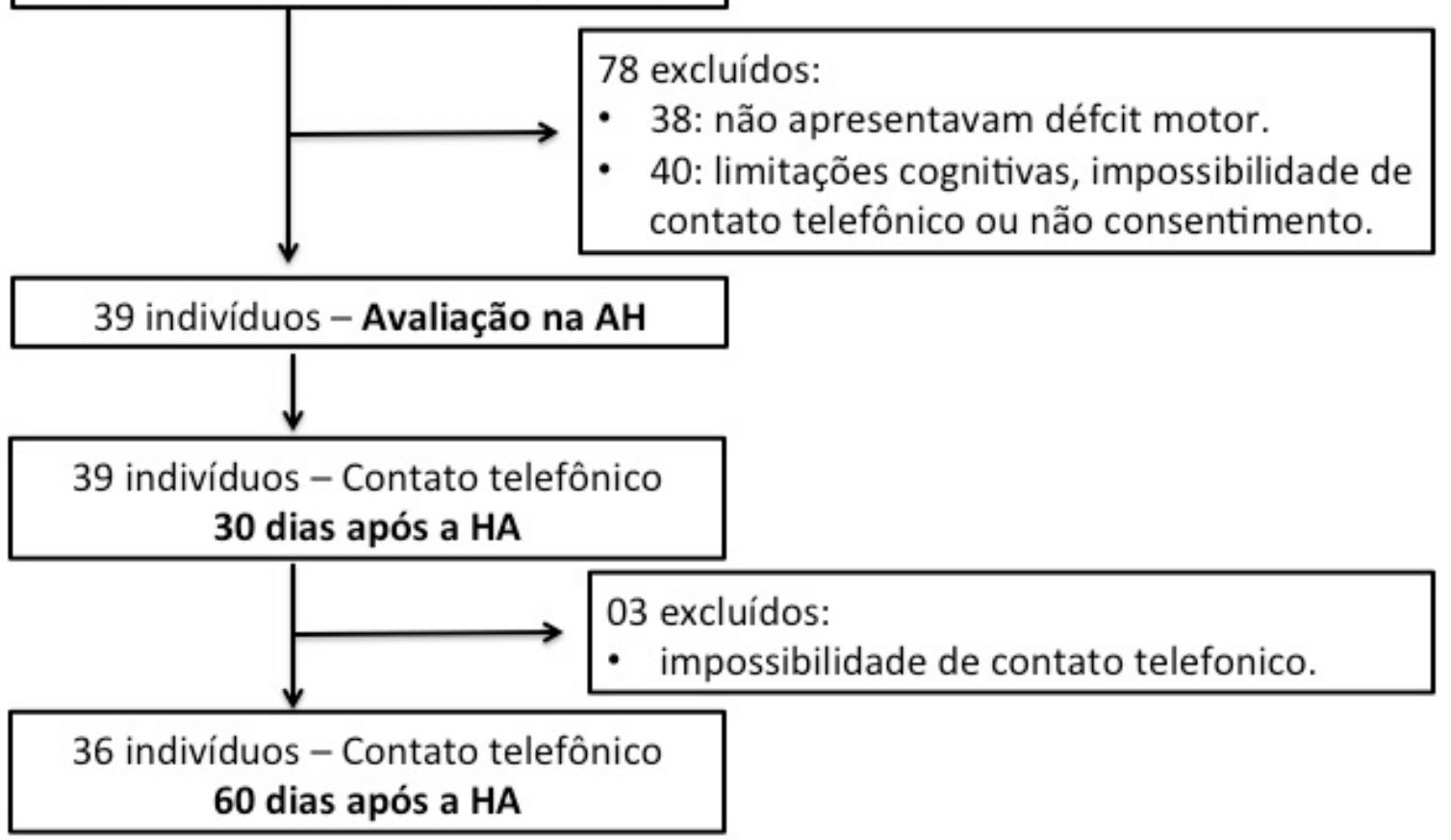

Figura 1: Fluxograma de coleta de dados. AVC: Acidente Vascular Cerebral; AH: Alta Hospitalar.

Tabela 1: Características da amostra.

\begin{tabular}{lc}
\hline \multicolumn{1}{c}{ Características da amostra } & $\mathbf{n}=\mathbf{3 6}$ \\
\hline Idade (anos) & $61 \pm 11$ \\
Sexo masculino & $22(61)$ \\
Cor branca & $30(83)$ \\
Procedência & \\
$\quad$ Região Metropolitana de Porto Alegre & $32(89)$ \\
Estado Civil & \\
Casado & $6(17)$ \\
Solteiro & $8(22)$ \\
Viúvo & $15(42)$ \\
Com quem residia antes do AVC & \\
Familiares (cônjuge e/ou filhos) & $28(78)$ \\
Sozinho & $7(19)$ \\
Instituições & $1(3)$ \\
Comorbidades & \\
Sedentarismo & $33(92)$ \\
Hipertensão Arterial Sistêmica & $31(86)$ \\
Tabagismo & $75(72)$ \\
Etilismo & $15(42)$ \\
Cardiopatia Isquêmica & $15(42)$ \\
Diabete Mellitus & $11(30)$ \\
Dislipidemia & $8(22)$ \\
Tempo de internação hospitalar (dias) & $15 \pm 2$ \\
\hline AVC: Acidente Vascular Cerebral. Valores expressos em média \pm \\
desvio padrão e frequência (\%).
\end{tabular}

Todos os indivíduos incluídos apresentavam déficit motor no momento da $\mathrm{AH}$, destes, 28 (78\%) realizaram acompanhamento fisioterapêutico durante a internação hospitalar. Somente 18 pacientes $(50 \%)$ receberam encaminhamento para fisioterapia na $\mathrm{AH}$ e $28(78 \%)$ relataram não estar organizados para realizar a fisioterapia após a alta. O tempo médio para o início da fisioterapia após a alta foi de 36 dias. A tabela 2 apresenta aspectos relacionados ao acesso a fisioterapia após a AH.

Sessenta dias após a $\mathrm{AH}$ apenas $39 \%$ dos pacientes estavam realizando fisioterapia. Destes, a maioria $(71 \%)$ frequentava a reabilitação em serviços públicos, disponibilizados pelo Sistema Único de Saúde (SUS). Entre os pacientes que não estavam realizando fisioterapia, a principal barreira apontada foi a dificuldade de acesso (95\%) aos serviços de reabilitação, seja por falta de encaminhamento, ausência de vagas ou burocracias para o início do acompanhamento.

Houve melhora na condição funcional da amostra estudada demonstrada através da variação no escore total da MIF e Rankin, 60 dias após a AH. Os valores da avaliação de funcionalidade (MIF) e incapacidade pós AVC (Rankin) no momento da AH e após sessenta dias são demonstrados na tabela 3. 
Quando comparamos os pacientes que realizaram fisioterapia após a AH com os que ainda não haviam iniciado a reabilitação, ambos os grupos apresentaram melhora funcional avaliada pela escala MIF conforme demostrado na figura 2 e tabela 3 . O grupo de indivíduos que realizou fisioterapia (após a $\mathrm{AH}$ ), no momento da $\mathrm{AH}$ apresentava um escore na escala MIF significativamente menor, comparado ao grupo que não realizou fisioterapia. Portanto, esses indivíduos eram piores funcionalmente no momento da AH. Após 60 dias, a diferença entre os grupos não permaneceu estatisticamente significativa, sugerindo efeito benéfico da fisioterapia na melhora funcional dos indivíduos estudados.

Tabela 2: Seguimento da reabilitação após a alta hospitalar.

\begin{tabular}{lcc}
\hline \multicolumn{1}{c}{ Reabilitação } & $\mathbf{3 0}$ dias & $\mathbf{6 0}$ dias \\
\hline Realizando Fisioterapia & $7(19)$ & $14(39)$ \\
Local onde realiza a reabilitação & & \\
$\quad$ Serviços Privados & $3(43)$ & $4(29)$ \\
$\quad$ Serviços de referência para o SUS & $4(57)$ & $10(71)$ \\
Forma de deslocamento & & \\
Transporte privado & $4(57)$ & $9(64)$ \\
Transporte coletivo & $3(43)$ & $5(36)$ \\
Frequência da fisioterapia (vezes/ & & \\
semana) & $3(43)$ & $2(15)$ \\
$\quad$ Uma vez & $4(57)$ & $12(85)$ \\
$\quad$ Duas vezes & & \\
Barreiras para o início da reabilitação & $20(69)$ & $12(55)$ \\
$\quad$ Dificuldades burocráticas & $7(24)$ & $9(41)$ \\
Falta de vagas (em lista de espera) & $2(7)$ & $1(4)$ \\
$\quad$ Transporte & &
\end{tabular}

SUS: Sistema Único de Saúde. Valores expressos em: frequência (\%).

\section{DISCUSSÃO}

O AVC pode ocorrer em qualquer idade, mas o risco duplica em cada década após os 55 anos, sendo que $70 \%$ dos AVCs acontecem em pessoas com 65 anos ou mais ${ }^{10-12}$. Considerando o rápido e intenso envelhecimento da população brasileira, este fato torna-se cada vez mais impactante para a saúde pública ${ }^{13,14}$. Os indivíduos do presente estudo tinham em média 61 anos de idade, sendo a maioria do sexo masculino (61\%), viúvos, que residiam com familiares. Tais achados concordam com a literatura

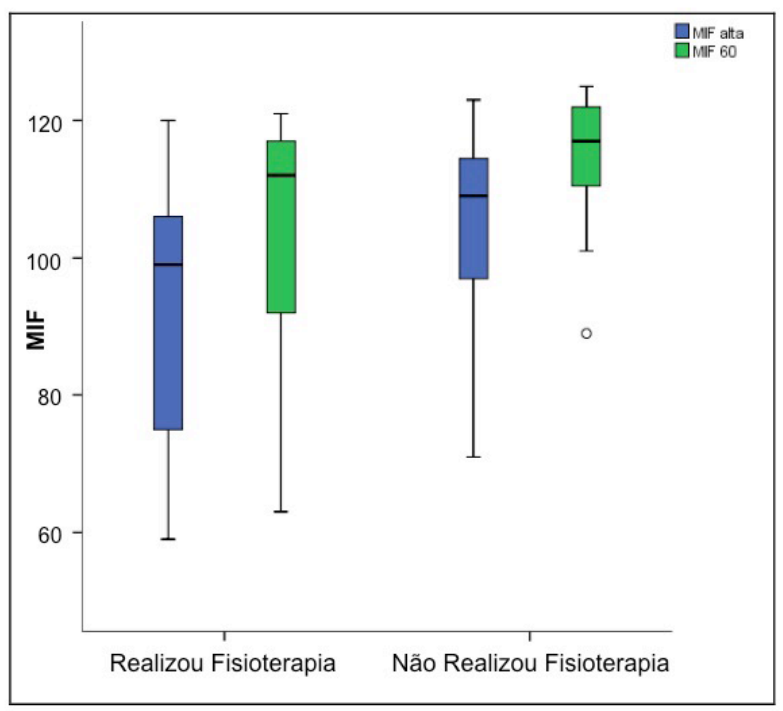

Figura 2: Comparação da Medida de Independência Funcional (MIF) na alta hospitalar e 60 dias após nos pacientes que realizaram ou não fisioterapia.

Tabela 3: Avaliação da funcionalidade após o Acidente Vascular Cerebral.

\begin{tabular}{|c|c|c|c|c|}
\hline Escalas & Alta & 60 dias & $p$ & \\
\hline MIF & $\begin{array}{c}105,50 \\
(94,3-112)\end{array}$ & $\begin{array}{c}115 \\
(107-119,5)\end{array}$ & 0,001 & \\
\hline MIF & & & & Delta \\
\hline Realizou fisioterapia & $\begin{array}{c}99 \\
(69-108,5)\end{array}$ & $\begin{array}{c}109 \\
(97-117)\end{array}$ & 0,001 & $\begin{array}{c}13,0 \\
(2,5-20,5)\end{array}$ \\
\hline Não realizou fisioterapia & $\begin{array}{c}112 \\
(90-117)\end{array}$ & $\begin{array}{c}117 \\
(110-123)\end{array}$ & 0,001 & $\begin{array}{c}7 \\
(2-13,0)\end{array}$ \\
\hline$p$ & 0,045 & 0,066 & & 0,415 \\
\hline
\end{tabular}

\section{Escala de Rankin}

0 - Sem sintomas

1 - Sem deficiência significativa

2 - Deficiência leve

3 - Deficiência moderada

4 - Deficiência moderada a grave

5 - Deficiência grave
0 (0)

12 (33)

$11(31)$

8 (22)

4 (11)
0,001

4 (11)

19 (53)

9 (25)

2 (6)

2 (6)

$0(0)$

MIF: Medida de Independência Funcional; Valores expressos em mediana (P25 - P75) e frequência (\%). 
revisada, que aponta para uma maior incidência de AVC em homens ${ }^{15}$, e com alta porcentagem de indivíduos que residem com outras pessoas (90\%), mais frequentes com cônjuge e filhos ${ }^{2}$.

Em relação aos fatores de risco para o AVC, encontramos taxas elevadas de hipertensão arterial sistêmica (HAS) (86\%), tabagismo (72\%) e sedentarismo (92\%). Nossos dados foram concordantes com a literatura que revela altas taxas de prevalência destes fatores de risco: HAS (64\%), tabagismo (60\%) e sedentarismo $(58 \%)^{10,16}$.

No Brasil, uma portaria publicada pelo Ministério da Saúde em 2012 regulamentou a linha de cuidado ao AVC. A mesma objetiva proporcionar cuidado integrado para esses pacientes com oferta de leitos hospitalares para reabilitação, adequada retaguarda ambulatorial para a assistência sequencial após a $\mathrm{AH}$, cuidados coordenados pelas equipes de atenção básica, reintegração social e ao trabalho, assim como o acesso de todos os pacientes às terapias estabelecidas em diretrizes, respeitando diferenças regionais ${ }^{3}$.

Quanto a reabilitação na fase hospitalar, observamos que $78 \%$ dos indivíduos realizaram acompanhamento fisioterapêutico durante a internação. Este fato provavelmente explica-se, pois, as unidades destinadas aos pacientes com AVC apresentam um número limitado de leitos, que ainda é inferior a real necessidade, e que a mesma assistência, quanto à reabilitação na fase hospitalar, nem sempre pode ser oferecida a todos os pacientes com AVC ${ }^{17}$. Resultados semelhantes foram observados em estudo realizado na Polônia, onde $80 \%$ dos pacientes receberam reabilitação precoce durante a internação em unidades de AVC e após eram internados em instituições dedicadas somente à reabilitação ${ }^{18}$.

Quanto ao seguimento da reabilitação após a $\mathrm{AH}$, passados 30 dias, apenas uma pequena porcentagem dos pacientes (19\%) estava realizando fisioterapia. Este fato pode ser parcialmente explicado pela baixa taxa de encaminhamento $(50 \%)$ recebido pelos pacientes no momento da $\mathrm{AH}$. Um estudo descritivo, realizado em Unidades de Saúde Comunitária de Porto Alegre, observou que a frequência de encaminhamentos, de pacientes em geral, à serviços de fisioterapia, foi extremamente baixa (1,1\%). Este fato foi atribuído a falta de confiança nas clínicas prestadoras de serviço, reduzindo o número de encaminhamento por parte dos médicos. Um fator limitante identificado foi a dificuldade em saber o que acontece com aqueles usuários encaminhados, se eles conseguem acesso à fisioterapia e se a mesma foi resolutiva ${ }^{19,20}$.

Um estudo recente realizado na cidade de João Pessoa mostrou que apesar da presença de comprometimento funcional, apenas $30,8 \%$ dos indivíduos tinham acesso aos serviços de fisioterapia após três meses do acometimento ${ }^{21}$. Outro estudo observou que $26 \%$ das pessoas com AVC nunca tiveram acesso a fisioterapia, mesmo passados 5,5 anos do evento ${ }^{22}$. Esses dados revelam uma difícil realidade recorrente no sistema de saúde brasileiro.

A não realização da fisioterapia ambulatorial pelo SUS está principalmente relacionada às dificuldades de acesso a estes serviços ${ }^{23}$. Uma das barreiras encontradas, no presente estudo, foi o longo tempo de espera. Após 60 dias $41 \%$ dos pacientes, que ainda não estavam realizando fisioterapia, aguardavam pelo serviço em uma lista de espera. Segundo Ferrer et al. (2015) a falta da organização da rede de serviços repercute em uma longa lista de espera para o atendimento fisioterapêutico ${ }^{24}$.

O tempo médio para o início da fisioterapia após a $\mathrm{AH}$ foi de 36 dias. Os resultados observados no presente estudo são semelhantes aos encontrados por Silva et al. (2014), onde os pacientes do município de Campo Grande levaram um tempo médio de 39 dias para iniciar o atendimento fisioterapêutico ${ }^{23}$. Esse tempo de espera pode postergar a recuperação, pois os estímulos precoces são fundamentais após o AVC ${ }^{25}$.

Evidencias apontam que o ideal é iniciar o tratamento imediatamente após o acometimento, visando potencializar a reorganização do tecido cerebral, maximizando assim a funcionalidade do paciente em suas atividades de vida diária ${ }^{26}$. $\mathrm{O}$ início tardio da intervenção fisioterapêutica pode ocasionar o aparecimento de contraturas e deformidades, dores, diminuição da força muscular e demora na reaprendizagem motora, dificuldades no equilíbrio e na marcha 4 .

Diversos estudos encontraram como limitações ao acompanhamento em serviços fisioterapêuticos ambulatoriais, na saúde pública, a dificuldade de acesso, o longo tempo de espera pela assistência, as questões financeiras e burocráticas de encaminhamento e agendamento que interferem na continuidade do tratamento, a quantidade limitada de atendimentos e a dificuldade de deslocamento até o local ${ }^{23-26}$. Silva et al. (2014) aponta a dificuldade de locomoção física dos pacientes que apresentam deficiência motora como um limitante para o acesso aos serviços públicos de reabilitação, já que a maioria dos usuários dependem do transporte público ${ }^{23}$.

A oferta de serviços de fisioterapia pelo SUS ainda é insuficiente, necessitando mais investimentos, seja por meio da contratação de recursos humanos, ampliação da rede de serviços ou disponibilização de transporte adequado para os usuários ${ }^{23}$. O Acesso aos serviços especializados, dentre eles a reabilitação, 
tem sido considerada um dos principais obstáculos para a efetivação da integralidade do SUS ${ }^{27}$.

Quanto a severidade dos déficits, 13 indivíduos (36\%) apresentavam deficiência moderada a grave no momento da $\mathrm{AH}$. Após 60 dias esse número passou para 4 indivíduos (12\%) com deficiência moderada e nenhum com deficiência grave, caracterizando claramente a melhora funcional do grupo estudado. A importância da fisioterapia após o AVC tem sido enfatizada na literatura, pois influencia na dependência e capacidade funcional dos indivíduos acometidos pelo AVC ${ }^{28,29}$. Benvegnu et al. ${ }^{30}$ observaram que os pacientes que prosseguiam com tratamento fisioterapêutico após a $\mathrm{AH}$ apresentaram uma diferença de 1,39 na média final da MIF, passando de dependente modificado para independente modificado. E os que não prosseguiam tiveram 0,19 de diferença na média final, mantendo a mesma categoria ${ }^{30}$.

O início precoce (nos primeiros seis meses) da reabilitação dos sujeitos após o AVC ganha destaque por ser possível, nesse período, se alcançar melhores respostas ao tratamento, com consequente redução das repercussões negativas na capacidade funcional ${ }^{26,31}$. A intensidade do tratamento, o período de tempo entre a lesão e o início da reabilitação são fatores que influenciam a recuperação da função neurológica ${ }^{32}$. A assistência fisioterapêutica tem um papel de destaque no contexto da reabilitação por contribuir principalmente para a prevenção de complicações, melhora do estado funcional e colaborar na adaptação às limitações decorrentes do AVC. Esses fatores impactam diretamente na reinserção dos indivíduos. Considerando-se os serviços envolvidos no processo de reabilitação, pesquisas verificaram que a abordagem fisioterapêutica tem sido a mais demandada pelos pacientes acometidos por $\mathrm{AVC}^{33}$.

Os serviços de reabilitação no Brasil, trabalham principalmente com pacientes ambulatoriais e esse acesso geralmente ocorre em períodos tardios ${ }^{34}$.
Diferente das fontes de literatura de outros países, onde a reabilitação ocorre predominantemente na fase aguda e em ambiente intra-hospitalar, como na Polônia que a reabilitação precoce era fornecida em unidades hospitalares ${ }^{17}$. Segundo Mizrahi (2015), pacientes pós AVC eram internados no Departamento de Reabilitação Geriátrica, onde se utilizava abordagem de equipe interdisciplinar que se reuniam duas vezes por semana para avaliar o estado de cada paciente, os mesmos passavam por uma média de cinco horas de fisioterapia por semana ${ }^{35}$.

Outra dificuldade encontrada pelos usuários que buscam o tratamento fisioterapêutico na rede pública é o número limitado de sessões de fisioterapia. Atualmente, na regulação do acesso, preconizam-se dez sessões, desconsiderando as particularidades do sujeito, sendo este número limitado, na maioria das vezes insuficiente. Além disso, ainda há a dificuldade na renovação dessas sessões, pois os pacientes necessitam retornar ao médico e recomeçar o fluxo do encaminhamento, o que retarda a continuidade do tratamento ${ }^{23}$.

O presente estudo apresenta algumas limitações como tamanho pequeno da amostra e o tempo limitado de acompanhamento aos indivíduos após a $\mathrm{AH}$, pois muitos ainda aguardavam pelo atendimento fisioterapêutico durante o contato telefônico nos 60 dias. Sugerimos a realização de estudos que possam acompanhar a longo prazo esses pacientes.

$\mathrm{O}$ acesso à fisioterapia após a $\mathrm{AH}$ para os indivíduos acometidos pelo AVC ainda é restrito. Diversas barreiras são encontradas para utilização desses serviços sendo a principal delas a dificuldade de ingresso aos serviços e o longo tempo de espera para iniciar o tratamento fisioterapêutico.

\section{Conflitos de Interesse}

Os autores declaram não haver conflito de interesse.

\section{REFERÊNCIAS}

1. Costa FA, Silva DL, Rocha VM. Estado neurológico e cognição de pacientes pós-acidente vascular cerebral. Rev Esc Enferm USP. 2011;45(5):1083-8. http://dx.doi.org/10.1590/S008062342011000500008. PMid:22031367.

2. Santos EB, Rodrigues RA, Marques $S$, Pontes-Neto OM. Estresse percebido nos idosos sobreviventes do AVC após a alta hospitalar para casa. Rev Esc Enferm USP. 2015;49(5):797803. http://dx.doi.org/10.1590/
S0080-623420150000500013. PMid:26516750.

3. Brasil. Ministério da Saúde. Linha de Cuidados em Acidente Vascular Cerebral (AVC) na rede de Atenção às Urgências e Emergências. Brasília: Ministério da Saúde; 2012.

4. Piassaroli CAP, Almeida GC, Luvizotto JC, Suzan ABBM. Modelos de reabilitação fisioterápica em pacientes adultos com sequelas de AVC isquêmico. Rev. Neurocienc. 2012;20(1):128-37.
5. Venna VR, Xu Y, Doran SJ, Patrizz A, McCullough LD. Social interaction plays a critical role in neurogenesis and recovery after stroke. Citation: Transl Psychiatry. 2014; 4(e351).

6. Sousa ARB, Ribeiro KSQS. A rede assistencial em fisioterapia no município de João Pessoa; uma análise a partir das demandas da atenção básica. $\mathrm{R}$ Bras Ci Saúde. 2011;15(3):357-368.

7. Brucki SMD, Nitrini R, Caramelli $\mathrm{P}$, Bertolucci PHF, Okamoto IH. 
Sugestões para o Uso do Mini Exame do Estado Mental no Brasil. Arq Neuropsiquiatr. 2003;61(3B):77781. http://dx.doi.org/10.1590/ S0004-282X2003000500014. PMid:14595482.

8. Riberto M, Miyazaki MH, Jucá SS, Sakamoto H, Pinto PP, Battistella LR. Validação da versão brasileira da Medida de Independência Funcional. Acta Fisiatr. 2004;11(2):72-6.

9. Brito RG, Lins LCRF, Almeida CDA, Neto ESR, Araújo DP, et al. Instrumentos de Avaliação Funcional Específicos Para o Acidente Vascular. Rev Neurocienc. 2013;21(4):593-9.

10. Costa VSP, Guimarães PSR, Fernandes KBP, Probst VS, Marquez AS, Fujisawa DS. Prevalence of risk factors for the occurrence of strokes in the elderly. Fisioter Mov. 2014;27(4):555-63. http://dx.doi. org/10.1590/0103-5150.027.004. AO07.

11. Carmo JF, Morelato RL, Pinto HP, Oliveira ERA. Disability after stroke: a systematic review. Fisioter Mov. 2015;28(2):407-18. http://dx.doi. org/10.1590/0103-5150.028.002. AR02.

12. Pereira $A B$, Alvarenga $H$, Pereira RS JR, Barbosa MT. Prevalência de acidente vascular cerebral em idosos no Município de Vassouras, Rio de Janeiro, Brasil, através do rastreamento de dados do Programa Saúde da Família. Cad Saude Publica. 2009;25(9):192936. http://dx.doi.org/10.1590/ S0102-311X2009000900007. PMid: 19750380.

13. Paiva RS, Valadares GV. Vivenciando o conjunto de circunstâncias que influenciam na significação da alta hospitalar: Estudo de Enfermagem. Esc Anna Nery. 2013;17(2):249-55. http://dx.doi.org/10.1590/S141481452013000200007.

14. Makiyama TY, Battisttella LR, Litvoc J, Martins LC. Estudo sobre a qualidade de vida de pacientes hemiplégicos por acidente vascular cerebral e de seus cuidadores. Acta Fisiatr. 2004;11(3):106-9.

15. Reeves MJ, Bushnell CD, Howard G, Gargano JW, Duncan P, Lynch $G$, et al. Sex diferences in stroke: epidemiology, clinical presentation, medical care, and outcomes.
Lancet Neurol. 2008;7(10):915-26. http://dx.doi.org/10.1016/S14744422(08)70193-5. PMid:18722812.

16. Castro JAB, Epstein MG, Sabino GB, Nogueira GLO, Blankenburg C, Staszko KF. Estudo dos principais fatores de risco para acidente vascular encefálico. Rev Bras Clin Med. 2009;7:171-3.

17. Veloso F, Reis LA, Azoubel R, Xavier TT, Argolo SM. Um olhar sobre a assistência fisioterapêutica a portadores de acidente vascular encefálico no município de Jequié BA. Rev Saúde. 2007;3(1):55-63.

18. Skibicka I, Niewada M, Skowrońska M, Członkowska A, Członkowski A. Care for patients after stroke. Results of a two-year prospective observational study from Mazowieckie province in Poland. Neurol Neurochir Pol. 2010;44(3):231-7. http://dx.doi. org/10.1016/S0028-3843(14)60036-0. PMid:20625958.

19. Silva GG, Sirena SA. Perfil de encaminhamentos a fisioterapia por um serviço de Atenção Primária à Saúde, 2012. Epidemiol Serv Saude. 2015;24(1):123-33. http://dx.doi.org/10.5123/S167949742015000100014.

20. Conill EM, Giovanella L, Almeida PF. Listas de espera em sistemas públicos: da expansão da oferta para um acesso oportuno? Considerações a partir do Sistema Nacional de Saúde espanhol. Cien Saude Colet. 2011;16(6):278394. http://dx.doi.org/10.1590/ S1413-81232011000600017. PMid:21709976.

21. Mendes LM, Gadelha DS, Brito GEG, Moraes RM, Ribeiro KSQS. Acesso de sujeitos pós-Acidente Vascular Cerebral aos serviços de fisioterapia. Revista de Enfermagem UFPE On Line. 2016;10(2):387-94.

22. Leite HR, Nunes APN, Corrêa CL. Perfil epidemiológico e qualidade de vida dos pacientes acometidos por Acidente Vascular Encefálico. Arq Ciênc Saúde UNIPAR. 2011;15(1):15-21.

23. Silva MA, Santos MLM, Bonilha LAS. Fisioterapia ambulatorial na rede pública de saúde de Campo Grande (MS, Brasil) na percepção dos usuários: resolutividade e barreiras. Interface Comunicacao Saude Educ. 2014;18(48):75-86.
24. Ferrer MLP, Silva AS, Silva JRK, Padula RS. Microrregulação do acesso à rede de atenção em fisioterapia: estratégias para a melhoria do fluxo de atendimento em um serviço de atenção secundária. Fisioter Pesqui. 2015;22(3):223-30.

25. Rangel ESS, Belasco AGS, Diccini S. Qualidade de vida de pacientes com acidente vascular cerebral em reabilitação. Acta Paul Enferm. 2013;26(2):205-12. http://dx.doi.org/10.1590/S010321002013000200016.

26. Huang HC, Chung KC, Lai DC, Sung SF. The impact of timing and dose of rehabilitation delivery on functional recovery of Stroke patients. J Chin Med Assoc. 2009;72(5):257-64. http://dx.doi.org/10.1016/S17264901(09)70066-8. PMid:19467949.

27. Spedo SM, Pinto NRS, Tanaka OY. O difícil acesso a serviços de média complexidade do SUS: o caso da cidade de São Paulo, Brasil. Physis. 2010;20(3):953-72. http://dx.doi.org/10.1590/S010373312010000300014.

28. Cecatto RB, Almeida CIO. Planejamento da reabilitação na fase aguda após o acidente vascular encefálico. Acta Fisiatr. 2010;17(1):3743.

29. Fernandes MB, Cabral DL, Souza RJP, Sekitani HY, Salmela LFT, Laurentino GEC. Independência funcional de indivíduos hemiparéticos crônicos e sua relação com a fisioterapia. Fisioter Mov. 2012;25(2):333-41. http://dx.doi.org/10.1590/S010351502012000200011.

30. Benvegnu AB, Gomes LA, Souza CT, Cuadros TBB, Pavão LW, Ávila SN. Avaliação da medida independência funcional de indivíduos com seqüelas de acidente vascular encefálico (AVE). Rev Cienc Salud. 2008;1(2):71-7.

31. Brewer L, Horgan F, Hickey A, Williams D. Stroke rehabilitation: recent advances and future therapies. Q J Med. 2013;106(1):11-25. http:// dx.doi.org/10.1093/qjmed/hcs174. PMid:23019591.

32. Borella MP, Sacchelli T. Os efeitos da prática de atividades motoras sobre a neuroplasticidade. Rev Neurocienc. 2009;17(2):161-9. 
33. Ribeiro RSQS, Neves RF, Brito GEG, Morais JD, Lucena EMF, Medeiros JM, et al. Perfil de usuários acometidos por Acidente Vascular Cerebral adscritos à estratégia Saúde da Família em uma capital do nordeste do Brasil. Rev Bras Cien Saúde. 2012;16(supl. 2):35-44.
34. Polese JC, Tonial A, Jung FK, Mazuco R, Oliveira SG, Schuster RC. Avaliação da funcionalidade de indivíduos acometidos por Acidente Vascular Encefálico. Rev. Neurocien. 2008;16(3):175-8.

35. Mizrahi EH, Fleissig Y, Arad M, Adunsky A. Functional gain following rehabilitation of recurrent ischemic stroke in the elderly: Experience of a post-acute care rehabilitation setting. Arch Gerontol Geriatr. 2015;60(1):108-11. http://dx.doi. org/10.1016/j.archger.2014.08.013. PMid:25239513.

Recebido: 15 jul, 2018 Aceito: 26 set, 2018 